\title{
RESET
}

Recherches en sciences sociales sur Internet

\section{Espace urbain et stratification sociale}

Une lecture spatiale des inégalités sociales à l'heure d'Internet

Urban Space and Social Stratification: A Spatial Interpretation of Social

Inequalities in the Internet Era

\section{Margot Beauchamps}

\section{OpenEdition}

Journals

Édition électronique

URL : http://journals.openedition.org/reset/139

DOI : $10.4000 /$ reset.139

ISSN : 2264-6221

Éditeur

Association Recherches en sciences sociales sur Internet

Référence électronique

Margot Beauchamps, «Espace urbain et stratification sociale », RESET [En ligne], 1 | 2012, mis en ligne le 30 décembre 2012, consulté le 02 mai 2019. URL : http://journals.openedition.org/reset/139 ; DOI : $10.4000 /$ reset.139

Ce document a été généré automatiquement le 2 mai 2019.

(c) Association Recherches en sciences sociales sur Internet 


\title{
Espace urbain et stratification sociale
}

\author{
Une lecture spatiale des inégalités sociales à l'heure d'Internet \\ Urban Space and Social Stratification: A Spatial Interpretation of Social \\ Inequalities in the Internet Era
}

Margot Beauchamps

L'auteure tient à remercier Samuel Coavoux et Sébastien François, ainsi que les relecteurs anonymes, dont les remarques précieuses et constructives ont contribué à clarifier le propos de cet article.

\section{Introduction}

1 Au fondement de la géographie se trouve le constat de l'hétérogénéité fondamentale de l'espace, de l'inégale répartition des objets (physiques ou sociaux) à la surface de la terre. La question «Pourquoi observe-t-on cela ici plutôt qu'ailleurs? ? synthétise l'approche géographique qui découle de ce constat. Au sein des espaces urbains, ces irrégularités dans la répartition des objets expriment plus visiblement qu'ailleurs des disparités sociales. Les qualités physiques des lieux (aménités paysagères ou au contraire, handicaps) mais plus encore la sédimentation qu'opère le temps long de l'histoire des villes façonnent les différents espaces de la ville en renforçant l'hétérogénéité des sousensembles (les «quartiers») qui la constituent. Cette hétérogénéité se traduit par une valorisation économique et symbolique plus ou moins grande des différents lieux de la ville qui est une manifestation (notamment) de leur inégale dotation en ressources de toute nature et de leur inégale accessibilité1. Ces écarts de valorisation conduisent à une répartition différenciée dans l'espace urbain des différentes catégories sociales ${ }^{2}$. «L'effet de naturalisation qu'entrâne l'inscription durable des réalités sociales » dans l'espace, ce que Bourdieu désigne comme "l'espace social réifié» participe à l'inertie des représentations et des caractéristiques sociales des différents lieux de l'espace urbain 
(Bourdieu, 1993 : 252). En retour, cette inscription dans l'espace de la structure sociale (c'est-à-dire de sa hiérarchie) contribue à l'inertie de cette structure sociale. En d'autres termes, « l'espace compte ». Il « agit, même s'il n'agit pas seul » (François et al., 2002 : 356) dans la structuration des phénomènes sociaux. L'interrogation sur la dimension spatiale des inégalités sociales ou, plus précisément, sur le rôle de l'espace dans la structuration de ces inégalités spatiales fait l'objet d'un intérêt, non pas tout à fait nouveau, mais, à tout le moins, récemment renouvelé dans les sciences sociales (Ripoll \& Tissot, 2010 ; Ripoll \& Veschambre, 2005).

2 Alors que ce champ de réflexion à la fois fructueux et complexe s'ouvre et n'est pas prêt de se refermer, l'espace, en tant qu'objet de recherche en sciences sociales et en particulier en géographie, est traversé par des évolutions rapides qu'il est urgent de prendre en compte. L'essor des techniques de l'information et de la télécommunication ( $\left.\mathrm{TIC}^{3}\right)$, la diffusion massive d'Internet et de la téléphonie mobile bouleversent de manière fondamentale les contraintes spatiales et temporelles qui s'exercent sur les activités humaines. S'il émerge au sein de la discipline un noyau de plus en plus consistant de recherches dans le domaine de la "géographie des TIC », la structuration, encore timide, de ce noyau semble avoir exempté le reste des géographes d'une réflexion sur les effets des usages de la télécommunication sur la manière dont les activités humaines se déploient dans l'espace, interrogation qui est pourtant au cœur de la géographie (Beaude, 2008a).

Cet article propose d'aborder le débat sur la dimension spatiale des inégalités sociales en l'articulant avec le bouleversement des contraintes spatio-temporelles lié à l'essor de la télécommunication, et en particulier d'Internet. Il s'interroge sur la manière dont Internet, en redéfinissant les contraintes spatiales, modifie ou non le rôle de l'espace dans la structuration de la société en différents groupes dotés de capacités inégales. Je fais l'hypothèse qu'Internet modifie l'articulation entre espace et inégalités sociales en réduisant les contraintes spatiales et temporelles qui s'exercent inégalement sur les différents groupes sociaux. La première partie de cet article pose les termes du débat sur la dimension spatiale des inégalités sociales en affirmant que l'espace est une entrée pertinente pour analyser la structuration, la hiérarchie de la société. La deuxième partie montre d'abord comment l'éclatement des contraintes spatiales et temporelles est susceptible de modifier l'articulation entre espace et inégalités sociales, et présente ensuite la méthode et les terrains choisis pour vérifier cette hypothèse. La troisième partie, enfin, apporte des éléments de réponse à partir du travail de terrain mené auprès d'habitants de deux quartiers classés en zone urbaine sensible de l'agglomération de Saint-Étienne en lien avec leurs usages d'Internet mais aussi plus généralement leur mode d'accès aux ressources sociales.

\section{Entrer par l'espace pour analyser la stratification sociale}

4 Alors que les transformations du système productif et l'élargissement de la «classe moyenne » ont affaibli le concept de classe comme entrée pertinente pour l'analyse de la structure de nos sociétés, les sciences sociales, et la société française dans un même mouvement, portent une attention de plus en plus grande aux marquages sociaux de l'espace urbain. En témoignent la place prise dans l'espace médiatique par le " problème 
des banlieues » et l'importance des publications portant sur la ségrégation socio-spatiale, qui pourraient laisser penser que l'espace urbain matérialise désormais plus efficacement les lignes de fractures de la société que ne le fait le concept de classe.

\section{Trouver le bon curseur pour une approche spatiale de la question sociale}

5 L'intérêt renouvelé pour la manière dont les inégalités sociales s'inscrivent spatialement participe d'un mouvement des sciences sociales qui reconnaissent à l'espace une importance de plus en plus grande dans les réflexions qui animent les différentes disciplines. La géographie, longtemps importatrice d'idées élaborées par d'autres disciplines, se transformerait en discipline exportatrice sous l'influence de ce mouvement, désigné comme le "tournant spatial» et auquel B. Warf et S. Arias ont consacré un ouvrage, The Spatial Turn, paru en 2009 (Warf \& Arias, 2009). Dans le premier chapitre de cet ouvrage, E. Soja, critiquant la géographie marxiste qui peine à sortir du cadre de pensée qu'offrent les classes sociales, affirme que «les processus spatiaux façonnent la forme sociale tout autant que les processus sociaux façonnent la forme spatiale. [...] Les relations spatiales d'inégalité sont aussi importantes en théorie et dans la pratique politique que les relations de classe. » (Soja, 2009 : 21).

6 Si la plupart des géographes, et désormais plus généralement, des chercheurs en sciences sociales admettent que l'espace joue un rôle, dans la production des inégalités sociales, la complexité des manières d'agir de l'espace est au fondement d'une grande partie des recherches dans les études urbaines et sociales, qui cherchent à démêler la part qu'on peut attribuer, dans l'explication de la permanence de la hiérarchie sociale, à la localisation des individus, aux espaces qu'ils se sont appropriés et aux caractéristiques de ces espaces (densité et diversité des ressources dont ils sont dotés, représentations symboliques qui leurs sont associées, etc.). Les quartiers concentrant les populations défavorisées occupent par exemple une grande partie de ces recherches, depuis les premiers travaux de l'école de Chicago. En France, cette question s'est renouvelée avec l'émergence de la politique de la ville.

7 L'idée que l'espace urbain, par sa fragmentation, non seulement cristallise les inégalités sociales, mais aussi peut contribuer à les accentuer, a donné lieu à un faisceau de recherches dont on peut repérer quelques questionnements récurrents. Le débat sur l'existence d'«effets de quartiers" en est un, qui se focalise sur l'influence de l'environnement social et urbain sur la trajectoire de vie des individus en faisant l'hypothèse que «la concentration des ménages pauvres dans des quartiers isolés spatialement et socialement du reste de la société urbaine a pour effet de redoubler les conséquences de la pauvreté » du fait, notamment, de l'influence des pairs (Fol, 2005 : 82 ; Bacqué \& Fol, 2007). Dans le débat sur les effets de quartiers, le risque d'autonomiser le rôle de l'espace dans la structuration sociale a été souligné pour rappeler que l'espace n'est pas extérieur aux rapports sociaux. Dans un numéro programmatique sur la « dimension spatiale des ressources sociales », F. Ripoll et S. Tissot proposent de « ne plus considérer l'espace et la société comme deux réalités séparées, extérieures l'une à l'autre, mais au contraire de penser l'espace comme une dimension inhérente aux rapports sociaux: le social est toujours déjà spatial. A minima, il faut considérer qu'"effets de classe" et "effets de lieu" interagissent constamment » (Ripoll \& Tissot, $2010: 5$ ). 
Un deuxième ensemble de recherches s'intéresse à l'inadéquation entre la localisation des quartiers défavorisés et celle des ressources sociales (surtout de l'emploi), ainsi qu'aux difficultés à se mouvoir dans l'espace urbain pour les populations socialement fragiles. L'hypothèse du spatial mismatch est à l'origine de nombreuses recherches empiriques aux Etats-Unis (Kain, 2004). En France, l'organisation urbaine différant grandement de celle des villes américaines, le débat sur le spatial mismatch se pose en d'autres termes, mais les questions concernant la localisation des ressources et la mobilité des habitants des quartiers considérés comme prioritaires au titre de la politique de la ville suscitent un vif intérêt (L'Horty \& Sari, 2008). L'éloignement des centres, voire l'enclavement d'une partie des quartiers classés en "Zone Urbaine Sensible », la rareté des équipements publics et privés, des emplois disponibles, la faible mobilité de leurs habitants et la stigmatisation sociale dont font l'objet certains de ces quartiers, ont été dénoncés comme autant de facteurs d'accentuation de l'exclusion sociale.

9 La mise en œuvre en France d'un projet politique spécifiquement dédié à des quartiers identifiés comme cumulant les difficultés socio-économiques, et à ce titre considérés comme prioritaires, la Politique de la Ville ${ }^{4}$, a contribué à l'émergence d'un discours sur la « crise de la ville » comme révélateur des nouvelles tensions sociales. Certains y ont vu un déplacement de la question sociale vers la question urbaine (Donzelot, 1991): la stratification verticale de la société qui prévalait dans la société industrielle fondée sur des rapports d'exploitation laisserait place à une stratification sociale horizontale basée sur des rapports d'inclusion ou d'exclusion de la société salariale et dont la relégation des exclus dans des quartiers de "banlieues» serait la manifestation spatiale. C. BidouZachariasen (1997) critique l'analyse selon laquelle la crise de l'urbain constituerait un déplacement de la question sociale. Selon elle, cette analyse ignore le caractère explicatif des effets spatiaux qu'il reste à mettre en évidence: l'espace n'est pas une simple projection des rapports sociaux. Les rapports sociaux s'inscrivent par nature dans l'espace, qui joue un rôle dans leur production. Le bon curseur pour une approche spatiale de la question sociale se situe donc dans une perspective qui n'ignore pas la dimension spatiale des rapports sociaux sans pour autant réduire les dialectiques sociales à des luttes spatiales, et je propose dans la suite de cet article d'aborder cette dimension spatiale par le truchement de la notion d'accessibilité aux ressources sociales.

\section{La question de l'accessibilité aux ressources sociales}

10 Formuler la question de l'articulation entre espace et structuration sociale en termes d'accessibilité aux ressources sociales permet d'appréhender de manière concrète le rôle de l'espace dans le maintien des inégalités sociales. La question de l'accessibilité aux ressources n'épuise certes pas tous les aspects de la dimension spatiale des inégalités sociales. Qu'en est-il, par exemple, des représentations associées aux lieux de résidence qui jouent un rôle dans la manière dont sont perçus et se perçoivent les citadins? On pourrait parfaitement considérer la valeur symbolique des lieux comme une ressource sociale susceptible d'être mobilisée par les individus pour faire valoir un certain positionnement social, replaçant ainsi la problématique de la valeur symbolique des lieux dans un questionnement sur l'accessibilité aux ressources. Même quand la valeur symbolique d'un lieu est entachée de connotations négatives (quartiers stigmatisés), l'appartenance à un territoire peut être mobilisée comme une ressource, comme l'ont montré certains en s'appuyant sur la notion de « capital d'autochtonie» (Renahy, 2010) 
Sans entrer plus loin dans ce débat, cet article considérera que, si elle laisse de côté d'autres manières d'aborder la dimension spatiale des hiérarchies sociales, la question de l'accessibilité aux ressources constitue néanmoins une approche féconde pour l'observation pratique des mécanismes par lesquels les contraintes spatiales jouent sur le positionnement social des individus. Les ressources sociales désignent ici l'ensemble des éléments matériels et immatériels, mobiles ou immobiles que peuvent mobiliser les individus en vue d'acquérir ou de maintenir une position dans l'espace social : emploi, logement, éducation, information, ressources culturelles, loisirs, santé (et plus généralement tout service marchand ou non marchand), réseau social, familial, commerces, etc. Elles constituent pour un individu des moyens d'augmenter son capital (économique, social, culturel, symbolique, etc.) ou des moyens de réaliser des transactions entre les différentes sortes de capital.

11 De plus, l'approche par la notion d'accessibilité offre la possibilité d'intégrer dans le débat sur le rôle de l'espace dans le maintien des inégalités sociales les pratiques sociales permettant d'atteindre des ressources par d'autres moyens que la mobilité. Je pense ici tout particulièrement à l'utilisation de la télécommunication. Ceci suppose de considérer la notion d'accessibilité du point de vue des individus et non pas seulement de l'utiliser pour qualifier des lieux. Adopter la perspective des personnes dans la réflexion sur l'accessibilité permet de déplacer l'attention sur l'ensemble des ressources urbaines et sociales que les individus peuvent potentiellement atteindre et sur les conditions de cet accès. Ferreira et Batey (2007), qui soulignent l'intérêt théorique autant qu'opérationnel d'aborder la question de l'accessibilité aux ressources du point de vue des individus et non seulement du point de vue des lieux, insistent également sur l'aspect multidimensionnel de la notion d'accessibilité. En plus des conditions de déplacement offertes par le système de transport (lui-même complexe puisqu'il fait intervenir une multiplicité d'acteurs), la notion intègre de nombreuses autres dimensions: le coût économique de l'accès, la perception de l'effort à fournir, les compétences qu'ont les individus (pour se déplacer ou pour acquérir une ressource), le temps disponible pour l'acquérir, la connaissance fragmentaire qu'ont les personnes de l'existence des ressources disponibles et de leur localisation, etc.

12 À ces dimensions multiples de l'accessibilité, on peut ajouter le système de dispositions acquises, produit par des conditions particulières d'existence que Bourdieu désigne par le terme d'habitus. De la même manière que la connaissance fragmentaire qu'un individu a, non seulement de la localisation des ressources disponibles, mais aussi plus simplement de leur existence réduit la possibilité d'y accéder, les habitus limitent les dispositions à rechercher telle ou telle ressource. Cette dernière dimension de l'accessibilité aux ressources constitue sans aucun doute la plus difficile à objectiver.

On comprend que la plupart de ces dimensions sont subjectives, ce qui justifie d'adopter la perspective des personnes pour appréhender l'accessibilité dans toute sa complexité. L'approche par l'accessibilité selon la perspective des personnes est utile afin d'identifier pour quelles catégories de populations l'effort à fournir pour accéder à certaines ressources est trop élevé. Elle offre ainsi la possibilité d'analyser la stratification de la société à partir des difficultés d'accès à certaines ressources. Le rôle de l'espace est essentiel dans ce mécanisme de stratification sociale par l'inégale accessibilité aux ressources selon les personnes puisque les ressources sociales sont ancrées dans l'espace, même lorsqu'elles sont mobiles (comme les personnes constituant un réseau social). On peut cependant se demander si l'importance prise, avec l'émergence d'une «société de 
l'information » (Castells, 1996), par les ressources sociales immatérielles, et donc, moins soumises aux contraintes spatiales, ne sont pas susceptibles de modifier le rôle que joue l'espace dans cette stratification sociale. La possibilité offerte par Internet d'accéder à une multitude de ressources sociales en ligne ne réduit-elle pas l'importance de la dimension spatiale des inégalités sociales?

\section{Internet modifie-t-il le rôle de l'espace dans la structuration sociale?}

\section{La géographie à l'épreuve d'Internet}

14 La large diffusion d'Internet dans la société française au cours de la dernière décennie pourrait introduire des éléments nouveaux dans le schéma d'interaction entre espace et inégalités sociales. En dotant potentiellement tout lieu connecté, ou point d'accès au réseau, des mêmes ressources numériques - c'est-à-dire l'ensemble des données numérisées contenues dans la totalité des serveurs dans le monde reliés entre eux par le protocole IP - Internet introduit une inflexion majeure dans l'hétérogénéité fondamentale des lieux. En tant que réseau, Internet vise l'idéal d'isotropie qui caractérise le concept de réseau (Dupuy, 1987) et comme outil de télécommunication, il est une réponse au problème de la distance, " c'est-à-dire l'existence d'un écart entre les différents éléments qui concourent à la vie sociale » (Lévy, 1994 : 65). Pour Jacques Lévy, la télécommunication est une manière de gérer le problème de la distance, au même titre que la ville en tant que configuration spatiale minimisant la distance entre les différents objets de la société, ou que la mobilité.

La notion de distance, «obstacle à l'interaction sociale» (Beaude, 2008b: 126), est l'expression la plus synthétique du problème posé par l'espace aux sociétés humaines. Lévy identifie trois manières de gérer ce problème de la distance à différentes échelles de temps: la mobilité des hommes, la télécommunication (le transfert d'information à distance), et la coprésence, c'est-à-dire la présence en un même lieu. Ce dernier mode de gestion de la distance peut être recherché par des formes d'organisation spatiale qui maximisent la densité (des hommes et des objets sociaux) et la diversité, deux notions qui sont au fondement de la définition de la ville qu'il propose. Il rejoint sur ce point Paul Claval pour qui « la ville est une organisation destinée à maximiser l'interaction sociale » (1981 : 4). En définissant la ville par le couple densité/diversité, Lévy introduit l'idée que l'espace peut se comprendre comme un continuum depuis des formes alliant la densité et la diversité maximales vers des formes de moindre densité et diversité, dessinant ainsi un gradient d'urbanité. Pour lui, les acteurs sociaux opèrent continuellement des arbitrages entre les différentes modalités de gestion de la distance, également désignées comme des «techniques de l'espace». Ainsi, résider dans un lieu de faible urbanité peut être compensé par une forte mobilité et/ou un recours fréquent à la télécommunication pour accéder aux ressources qui manquent sur place, notamment les occasions d'interactions sociales.

16 En tant que réseau et en tant qu'outil de télécommunication, «technique de l'espace ", Internet pourrait être de nature à modifier la manière dont l'espace agit sur la structuration sociale. En permettant l'accès aux mêmes ressources numériques depuis l'ensemble des points d'entrée du réseau, Internet pourrait réduire l'écart qui sépare les quartiers disposant de ressources nombreuses et variées et ceux marqués par des 
faiblesses socio-économiques et par une faible urbanité (faibles diversité et densité d'objets), réalisant ainsi en partie le potentiel d'isotropie propre aux réseaux. La réduction de cette inégalité territoriale par l'homogénéisation des ressources disponibles dans les différents lieux pourrait alors se traduire par une réduction des inégalités de chances dont disposent les individus.

Cependant, de la même manière que la position sociale des individus détermine, d'une part, leur mode d'appropriation des différents espaces urbains, dont le lieu de résidence est le signe le plus évident, et, d'autre part, leur capacité à se déplacer (qui contribue d'ailleurs au niveau d'appropriation des espaces urbains), les individus ne se trouvent pas égaux devant la manière de tirer parti des avantages offerts par Internet. Comme les chiffres les plus récents en France (CREDOC, 2011) le confirment, les inégalités d'accès aux ressources de la société de l'information recoupent essentiellement les inégalités sociales existantes. La position sociale des individus et leur capital économique, social, culturel déterminent fortement les capacités d'accès aux ressources d'Internet par le jeu complexe des multiples sources de différenciations en matière de niveau d'accès aux ressources numériques. Les divers indicateurs du niveau d'appropriation d'Internet (l'équipement en ordinateur, en connexion Internet et outils de télécommunication en général, et le degré d'usage d'Internet en termes de fréquence comme en termes de variété de pratiques en ligne) convergent pour mettre en évidence l'importance de l'âge et des capitaux social, culturel et économique comme déterminants pour la capacité des individus à s'emparer de l'opportunité que représente Internet.

\section{À portée du centre, à portée de clic : deux quartiers prioritaires de l'agglomération stéphanoise}

18 Pour confronter à l'épreuve du terrain l'hypothèse de la capacité d'Internet à modifier les mécanismes de stratification sociale, l'analyse qui suit s'appuie sur 45 entretiens auprès d'habitants de deux quartiers de l'agglomération de Saint-Étienne classés en ZUS (zone urbaine sensible). Les entretiens, menés auprès de personnes d'âges variés (de 15 à 88 ans) en situation de faibles capacités socio-économiques (précarité financière, difficulté d'accès à l'emploi, parfois en raison d'un faible niveau de maitrise de français et/ou de l'écrit, et de manière générale d'un faible niveau d'éducation), visaient à retracer l'histoire individuelle d'appropriation d'Internet à travers les trajectoires résidentielle, professionnelle, et plus généralement la trajectoire sociale de la personne interrogée ${ }^{6}$. Il s'agissait également de relier les usages d'Internet avec les pratiques de mobilité ainsi que les projets individuels à différentes échelles de temps. Les entretiens cherchaient enfin à identifier pour les non-usagers d'Internet, ou pour les personnes ayant un usage limité d'Internet (en fréquence d'utilisation ou en types de pratiques), les contraintes qui s'exerçaient. Je me suis donc intéressée tant aux personnes qui n'avaient jamais utilisé Internet qu'aux internautes réguliers (avec entre les deux, toute une gamme de modes d'utilisation).

L'hypothèse ayant présidé à la conception de la grille d'entretien était que les difficultés d'accès aux ressources de la société d'information peuvent être d'autant plus problématiques que l'on réside dans un quartier peu accessible, c'est-à-dire à partir duquel il est plus difficile d'accéder aux ressources que sont l'emploi, les loisirs, les services, les commerces, etc. À l'inverse, les ressources offertes par Internet peuvent tempérer dans les quartiers périphériques la difficulté d'accès aux ressources urbaines. 
Figure 1 : Localisation des quartiers

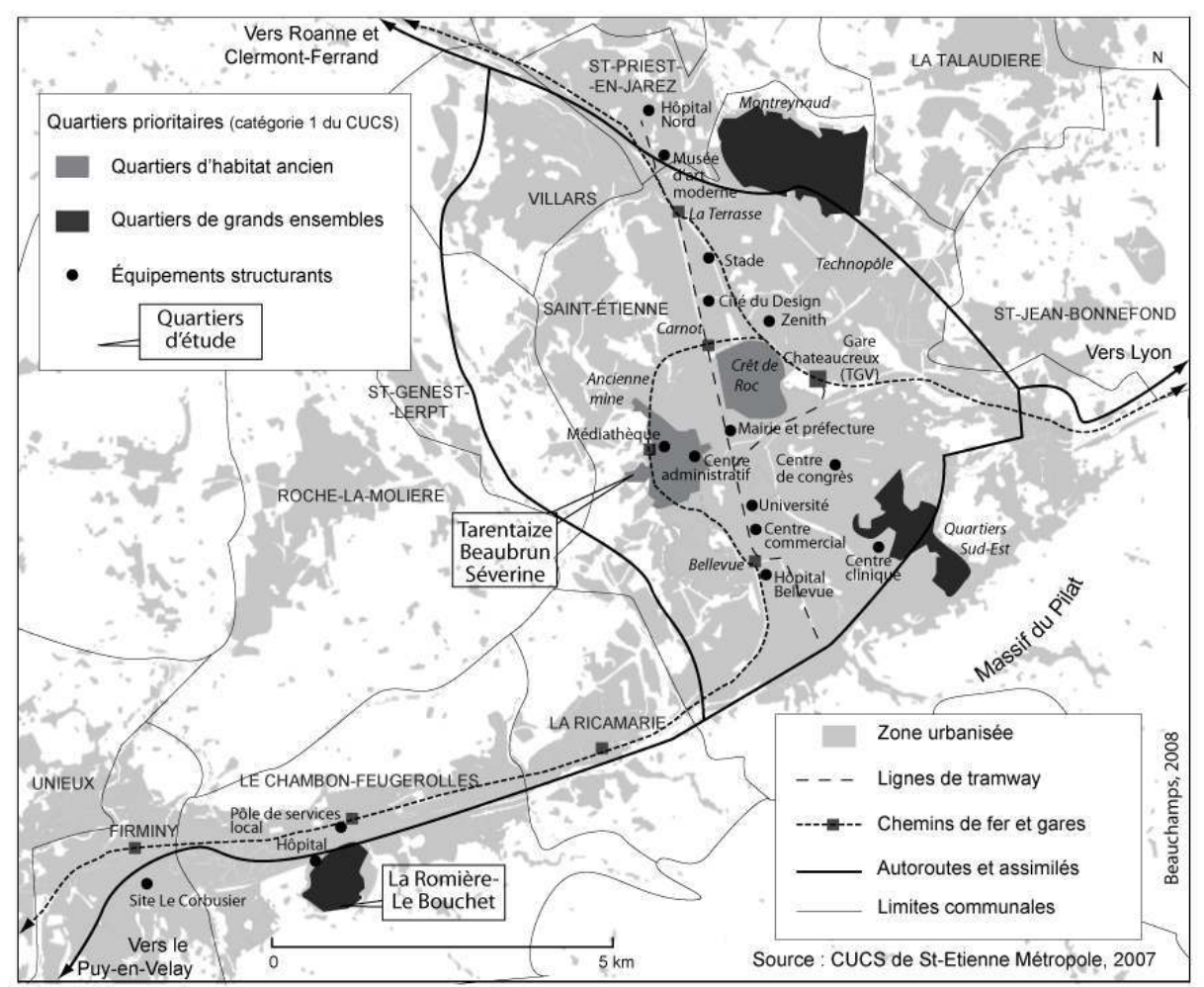

Pour interroger cette hypothèse, les entretiens ont été menés dans deux quartiers comparables en ce qui concerne leur structure sociale (voir Tableau 1), mais très différents quant à leur situation urbaine (voir Figure 1). Ils font tous deux partie des cinq quartiers prioritaires au titre de la catégorie 1 (catégorie qui rassemble les quartiers les plus en difficulté) du Contrat Urbain de Cohésion Sociale (CUCS) de l'agglomération?

Le quartier de Tarentaize-Beaubrun (TBS), à quelques centaines de mètres de l'hypercentre de la ville, est un quartier d'habitat ancien qui a bénéficié des dispositifs mis en œuvre dans le cadre de la Politique de la Ville depuis l'origine. Sa proximité avec l'ancienne mine de charbon principale de la ville en a fait un quartier ouvrier et d'immigration dès le xix ${ }^{\text {ème }}$ siècle. La fermeture progressive des puits de la mine au cours des années 1960 et 1970 et plus généralement de nombreuses usines qui avaient contribué à faire de Saint-Étienne une ville industrieuse du XIXème siècle jusqu'aux années 1970 ont transformé les structures sociales du quartier organisées autour du travail ouvrier et placé une grande partie de sa population, faiblement qualifiée, au chômage. Dans le paysage, ces difficultés socio-économiques se traduisent par une dégradation de l'habitat, la lutte contre le logement insalubre étant l'un des axes essentiels de l'action publique locale. Le quartier bénéficie de nombreux commerces et services de toute nature. La principale médiathèque de la ville est implantée au cœur du quartier qui dispose également d'une mairie annexe et d'un centre administratif, ainsi que des équipements sportifs et de loisirs gérés par le secteur associatif. Ces ressources urbaines denses et variées contribuent à diversifier la population qui se rend régulièrement dans le quartier. Si l'on ne peut parler de gentrification (la structure sociale du quartier restant essentiellement caractérisée par la prédominance des ménages modestes, souvent en situation précaire), on compte parmi les habitants du quartier une (très) petite partie de 
ménages aux revenus stables, et plus élevés (souvent plus âgés) ou encore des personnes jeunes aux revenus encore faibles mais au capital social et culturel élevés, laissant présager une augmentation des revenus au fil de leur intégration dans le monde du travail.

La Romière-Le Bouchet (LRLB) dans la commune du Chambon-Feugerolles est un quartier de grand ensemble dont la construction s'est étalée sur plusieurs décennies (1950 à 1970). Il est situé à une dizaine de kilomètres du centre-ville de Saint-Étienne, dans la vallée industrielle de l'Ondaine. Il est relativement peu accessible en transport en commun, et très peu équipé en services et commerces. LRLB, sur les hauteurs du versant sud de la vallée, se trouve de plus coupé du centre-ville du Chambon-Feugerolles par la voie de chemin de fer et la route nationale reliant Firminy à Saint-Étienne. Comme l'ensemble de l'agglomération stéphanoise, la vallée de l'Ondaine a souffert de nombreuses fermetures d'usines qui ont affecté les possibilités d'insertion professionnelle et sociale de ses habitants (voir Tableau 1). Le quartier de LRLB n'échappe pas à la tendance, générale à l'échelle de l'agglomération, d'un déclin démographique du fait d'un solde migratoire déficitaire. L'augmentation progressive du nombre de logements vacants dans le quartier a incité la municipalité, en accord avec les services de l'État, à orienter le programme de rénovation urbaine vers une démolition de logements sans reconstruction sur place mais dans le centre-ville de la commune. Cette décision a accéléré la disparition de certains petits commerces dans le quartier. Elle a aussi justifié la fermeture de certains équipements publics (comme la Maison des Jeunes et de la Culture), accentuant ainsi le déficit d'équipements pour les habitants restant sur le site.

Tableau 1 : Données sur la composition sociale des deux quartiers

\begin{tabular}{|l|l|l|l|}
\hline & $\begin{array}{l}\text { Quartier } \\
\text { TBS }\end{array}$ & $\begin{array}{l}\text { Quartier } \\
\text { LRLB }\end{array}$ & $\begin{array}{l}\text { Ensemble } \\
\text { l'agglo-mération }\end{array}$ \\
\hline $\begin{array}{l}\text { Part de la population de } 15 \text { ans et plus sans } \\
\text { aucun diplôme }\end{array}$ & $46 \%$ & $47 \%$ & $23 \%$ \\
\hline $\begin{array}{l}\text { Part des diplômés du supérieur parmi la } \\
\text { population de } 15 \text { ans et plus }\end{array}$ & $10 \%$ & $15 \%$ & $20 \%$ \\
\hline $\begin{array}{l}\text { Taux de chômage } \\
\text { Taux de chômage des 15-24 ans }\end{array}$ & $23 \%$ & $21 \%$ & $13 \%$ \\
\hline $\begin{array}{l}\text { Part des ouvriers dans l'ensemble de la } \\
\text { population active }\end{array}$ & $36 \%$ & $58 \%$ & $23 \%$ \\
\hline $\begin{array}{l}\text { Part des employés dans l'ensemble de la } \\
\text { population active }\end{array}$ & $30 \%$ & $25 \%$ & $30 \%$ \\
\hline $\begin{array}{l}\text { Part des Cadres et Professions Intellectuelles et } \\
\text { supérieures dans l'ensemble de la population } \\
\text { active }\end{array}$ & $7 \%$ & $4 \%$ & $12 \%$ \\
\hline
\end{tabular}


23 À partir des entretiens mais aussi d'une insertion dans la vie quotidienne de certains habitants de ces deux quartiers en tant que bénévole au sein d'associations ${ }^{8}$, ce travail de terrain effectué dans une démarche d'observation participante a permis d'identifier les manières de faire sans Internet de personnes en situation de disqualification sociale dans un contexte social de dépendance de plus en plus grande au numérique. La notion de dépendance numérique (directement empruntée à celle de dépendance automobile ${ }^{9}$ ) désigne une configuration sociale dans laquelle tout est conçu pour les usagers des TIC. La pénétration du numérique et d'Internet dans une grande partie des actes de la vie quotidienne s'accompagne bien d'une incitation de plus en plus forte à utiliser Internet pour accéder à un panier de ressources sociales toujours plus large. Les travaux d'Éric Dagiral sur le chantier mené par l'État et les administrations publiques pour la mise en place d'une administration électronique montrent que la "dimension technologique » prime sur les bienfaits attendus d'une modernisation de l'administration pour les usagers, suscitant des critiques et des appels à une meilleure articulation des portails en ligne avec « le guichet, le téléphone et le courrier » (Dagiral, 2011). Cette incitation s'exprime aussi par le biais du discours publicitaire, qui, sur les affiches comme à la télévision, vante des offres commerciales uniquement disponibles par le Web. Pour les familles comprenant des enfants scolarisés, cette incitation à utiliser Internet, ou plus précisément à se doter d'un ordinateur et d'une connexion à Internet à domicile pour les besoins scolaires des enfants, prend la forme, dès l'entrée dans le secondaire, d'une injonction, intégrée par les parents qui relaient le plus souvent la pression exprimée par leurs enfants, et parfois aussi par une partie des équipes pédagogiques. Enfin, le discours politique sur la «fracture numérique» et les instruments de sa mesure utilisés par les institutions publiques contribuent à alimenter l'idée qu'une partie de la population aurait un retard à rattraper et devrait s'aligner sur les pratiques des internautes, sans considération particulière pour ce que Fabien Granjon désigne comme la «conversion en accomplissement de "bien-être" des possibilités d'actions offertes par l'informatique connectée » (Granjon, 2011 : 68). Pour Éric Guichard, la création et la collecte de chiffres sur la «fracture numérique » légitiment le concept que ces statistiques sont censés évaluer : «le chiffre produit la catégorie plus qu'il n'en est la conséquence ou la mesure » avec pour objectif, plus ou moins prégnant, mais très rarement avoué, de soutien à l'industrie des TIC (Guichard, 2011).

24 Cette incitation sociale à utiliser Internet entre en contradiction avec les obstacles que rencontrent une partie de la population pour s'approprier les multiples pratiques rendues possibles par Internet: freins financiers, cognitifs, culturels. Il ne s'agit pas ici de proclamer l'avènement d'une telle configuration $d u$ monde, déjà universellement dépendante d'Internet, mais de poser l'hypothèse que cette tendance à la restructuration de plus en plus systématique des services (marchands ou non) pour s'orienter vers des usagers dont on suppose qu'ils sont internautes peut augmenter les difficultés d'accès à certaines ressources sociales pour une partie de la population, et partant accentuer les mécanismes de stratification sociale liés aux inégalités d'accès aux ressources. Ces difficultés sont-elles plus problématiques dans les quartiers faiblement équipés et à l'accessibilité réduite? 


\section{Une consolidation des positions sociales à l'heure d'Internet?}

Analyser les modalités de l'appropriation d'Internet par les personnes en situation de disqualification sociale (Paugam, 2000) en replaçant ces modalités dans le cadre plus général des trajectoires de vie présente un intérêt double quand il s'agit de comprendre les mécanismes de stratification sociale à l'œuvre aujourd'hui. D'une part, cela permet d'observer les dynamiques d'exclusion sociale ou au contraire de renforcement de la capacité à agir selon le niveau d'appropriation d'Internet. D'autre part, s'intéresser aux processus de stratification sociale liés à l'accessibilité aux ressources sociales permet d'enrichir le débat sur la dimension spatiale des inégalités sociales. Les pages qui suivent présentent d'abord quelques éléments de réponse à la question du rôle de la localisation des ménages socialement défavorisés sur les conditions d'accès aux ressources sociales et sur la place qu'y tient l'usage d'Internet. Puis seront présentées, dans les deux types de configuration urbaine étudiés, les stratégies développées par les personnes éloignées d'Internet pour s'en sortir dans ce contexte de dépendance numérique croissante.

\section{Un rôle marginal du degré d'urbanité dans le niveau de recours à Internet}

Les entretiens n'ont justement pas permis de vérifier une différenciation entre les deux quartiers concernant le niveau de recours à Internet pour accéder aux ressources dont les personnes enquêtées avaient besoin. Plusieurs raisons peuvent être avancées pour expliquer l'absence de différenciation marquée entre les deux quartiers étudiés en ce qui concerne le niveau de recours à Internet. Cet outil de télécommunication ne propose pas seulement un substitut à la mobilité pour certains déplacements autrefois inévitables, mais permet aussi et surtout d'accéder à un certain nombre de ressources qui étaient simplement indisponibles auparavant, ou au prix d'efforts tels que le renoncement était l'issue la plus fréquente. C'est en particulier le cas en ce qui concerne certaines informations culturelles ou scientifiques, ou encore le maintien de certaines relations (amicales ou non) que l'on peut classer parmi les liens faibles. Pour ce type de ressources, le degré d'urbanité des quartiers (entendue comme le degré de densité et diversité d'objets sociaux) ne semble pas une variable pertinente pour expliquer le besoin ou non d'un recours à Internet. Qu'il s'agisse des ressources pour lesquelles Internet est un substitut possible à la mobilité, ou de celles pour lesquelles Internet tend à devenir l'unique vecteur pour y accéder (services administratifs, d'offres commerciales, d'offre d'emploi ou de logement, etc.), on trouve dans les deux quartiers une proportion équivalente de personnes qui déploient des stratégies de substitution à l'usage d'Internet pour palier la marginalité dans laquelle elles se trouvent concernant l'usage ou l'accès à Internet.

Outre le degré d'accessibilité et d'équipement des quartiers, le degré d'urbanité au sens où l'entend Jacques Lévy (une combinaison de densité et de diversité) pourrait hypothétiquement influer sur le niveau de dépendance numérique des différents quartiers. En effet, du degré d'urbanité d'un quartier dépend sa capacité à offrir des opportunités d'interactions sociales diversifiées. Citant l'anthropologue Ulf Hannerz, Yves Grafmeyer souligne l'une des propriétés essentielles de la ville : «Si la vie urbaine 
favorise l'accessibilité mutuelle des êtres sociaux qui cherchent à entrer en contact, elle multiplie en même temps les occasions de rencontres non programmées. [...]: "L'accessibilité urbaine, aujourd'hui comme par le passé est partiellement planifiée mais aussi partiellement aléatoire. Bousculer quelqu'un qu'on n'a pas vu, assister à des scènes qu'on n'a pas prévues, voilà des expériences qui ne sont sans doute ni utiles ni agréables, mais qui ont peut-être des conséquences particulières sur le plan personnel ou sur le plan social et culturel [...]. Le flair (serendipity), le fait de découvrir quelque chose par hasard alors qu'on en cherchait une autre, est peut-être une aptitude que privilégie la vie urbaine". » (Grafmeyer, 1995 : 10).

Dans le quartier peu dense et sous-équipé de LRLB, on pourrait donc s'attendre à une plus grande dépendance à Internet que dans le quartier de Tarentaize en centre-ville de SaintÉtienne pour accéder à cette ressource urbaine qu'est la serendipity à travers le Web, et notamment les sites de rencontres (de type Meetic ou tchatche.com) et les sites de réseaux sociaux comme Facebook et Skyblog que certaines personnes interrogées utilisent pour nouer des relations virtuelles. Or, les entretiens ont montré qu'on trouve des internautes recherchant sur le Web cette fonction de serendipity que pourrait procurer l'espace urbain, aussi bien dans le quartier de centre-ville que dans le quartier périphérique. À position sociale, âge, situation familiale et position au sein de la famille comparables, les habitants de Beaubrun, qui disposent d'espaces de sociabilité plus nombreux et permettant des rencontres socialement plus diverses, sont aussi disposés que ceux de LRLB à consacrer une partie de leur temps sur des forums de chat ouverts à tous, ou bien sur des sites de réseaux sociaux comme Facebook ou Skyrock pour discuter depuis un profil public avec des inconnus, ce qui peut conduire à nouer de véritables liens d'amitié, débouchant ou non sur des rencontres physiques. Dans le même temps, les habitants des deux quartiers développent également des stratégies hors ligne pour s'exposer à des interactions sociales non programmées, ou seulement en partie programmées, à travers l'assignation d'un rôle de sociabilisation à certains lieux publics, et à certains horaires, qui diffèrent selon les âges et les sexes : une aire de jeu, un coin de rue avec quelques bancs, et bien évidemment dans le quartier de Tarentaize, les cafés, les commerces. Ainsi, les habitants de La Romière-Le Bouchet, quartier dépourvu de cafés et de commerces de proximité (à la notable exception d'une épicerie et d'une pharmacie situées en lisière du quartier) recréent des lieux identifiés par un groupe social aux limites définies ${ }^{10}$ comme espaces de sociabilité régulière, parfois quotidienne : il peut s'agir d'un banc où l'on sait que l'on a des chances de croiser une connaissance, sans avoir besoin de prendre rendez-vous, de la même manière que l'internaute se connectant à son compte MSN sait qu'à certaines heures, il aura des chances de trouver des connaissances connectées au même moment. On trouve dans les deux quartiers chez certaines personnes interrogées une logique de complémentarité entre sociabilité urbaine et sociabilité numérique; chez d'autres une logique de substitution de la sociabilité numérique à la sociabilité urbaine, (logique qui concerne surtout les personnes dont la mobilité est fortement contrainte ${ }^{11}$ ); chez d'autres encore une absence de sociabilité numérique (logique qui concerne, bien entendu, en premier lieu les non-internautes, mais aussi des internautes qui créditent de peu de valeur les échanges en ligne, ou encore des internautes dont le réseau social est faiblement structuré). Le rôle du degré d'urbanité du quartier de résidence sur cette pratique du Web comme lieu de démultiplication des rencontres possibles ne semble jouer qu'à la marge. Ce constat confirme que ces deux types de sociabilités ne répondent pas aux mêmes besoins d'interaction sociale. 


\section{S'en sortir dans un contexte de dépendance numérique croissante} en évidence par Sylvie Fol (2010) dans le contexte d'une exigence de flexibilité L'aisance dans le domaine des télécommunications devient une exigence de plus en plus
prégnante pour une réelle participation sociale et une insertion professionnelle réussie.
L'injonction à l'usage d'Internet semble aussi forte que l'« injonction à la mobilité » mise caractéristique du "nouvel esprit du capitalisme » (Boltanski \& Chiapello, 1999). Au-delà de l'insertion professionnelle, c'est la capacité à participer à la vie sociale qui est en jeu. Dans ce contexte, comment les personnes qui rencontrent un faisceau d'obstacles dans l'appropriation d'Internet parviennent-elle à accéder aux ressources sociales (indispensables ou désirées) nécessitant Internet tout en s'affranchissant du modèle de la dépendance numérique qui tend à s'affirmer par ailleurs? Parmi les personnes interrogées dans les deux quartiers stéphanois, une majorité était confrontée à divers freins (financier, culturel, cognitif, etc.) qui réduisent soit leur niveau d'équipement en micro-ordinateur et connexion Internet, soit leur capacité à maintenir en état de marche cet équipement, soit la valeur d'usage même d'une connexion à Internet par manque de compétences, ou même de connaissance des possibilités offertes par les applications Internet.

Pour ceux qui, bien qu'internautes réguliers (voire quotidiens), ne possèdent pas d'ordinateur, quand leur réseau social rapproché ne leur permettait pas de se connecter au domicile d'un proche, la dépendance numérique les amenait à fréquenter assidûment les cybercafés auxquels ils consacrent une part importante de leur budget. C'était le cas de trois demandeurs d'emploi interrogés, dont un dans le quartier de LRLB, qui se rendait dans le centre du Chambon-Feugerolles. Ceux-ci ne sortent donc pas du modèle de la dépendance numérique, puisque l'absence d'équipement ne limite qu'en partie l'usage d'Internet.

Les stratégies déployées par les non-usagers de l'Internet pour s'affranchir de la dépendance numérique sont en revanche d'une autre complexité. Cette catégorie des «non-usagers» n'est pas sans poser quelques difficultés de définition (Boutet \& Tremembert, 2008; Granjon, 2010). En effet, parmi les personnes interrogées, rares sont celles qui n'ont jamais eu affaire à Internet. Dans cette catégorie construite du nonusager, on trouve différents types d'éloignement par rapport à la pratique d'Internet: ainsi, au-delà des non-usagers absolus (c'est-à-dire ceux qui ne se sont jamais, au cours de leur vie, connectés à Internet), on trouve des usagers intermittents qui ont une utilisation très sporadique d'Internet, les abondonnistes, qui ont renoncé à utiliser Internet par manque de compétence, d'intérêt, parfois d'argent, ou encore ceux qu'A. Lenhart et al. (2003) appelle les «net-evaders » qui vivent dans un environnement où Internet est très présent mais qui font le choix de ne pas utiliser Internet seuls et déploient en conséquence des stratégies d'évitement.$$
\text { conséquence des stratégies d'évitement. }
$$ 
d'emploi sont consultables sur Internet et disparaissent peu à peu des autres vecteurs non numériques de diffusion. Ainsi, Pôle Emploi ne propose plus de classeur d'annonces, toutes les annonces étant désormais consultables soit à distance par Internet, soit sur les postes informatiques disponibles dans les agences. Dans ces conditions, quelles sont les alternatives pour les non-usagers? Outre les agences d'intérim, vers qui se tournent de nombreuses personnes interrogées qui y trouvent des emplois exigeant un faible niveau de qualification, le réseau social, familial, amical ou de voisinage reste, pour les personnes interrogées, le premier vecteur d'information sur les emplois disponibles. Les candidatures spontanées sous forme de déplacements auprès d'employeurs potentiels (restaurateurs, entreprises d'artisanat, commerces) sont également privilégiées par plusieurs des personnes interrogées.

En plus du recours au réseau social et aux déplacements de proximité, il faut souligner le rôle essentiel des travailleurs sociaux pour résoudre les difficultés d'accès aux ressources sociales recherchées par les personnes interrogées. Ceux-ci jouent souvent un rôle de médiateur, et deviennent l'interface entre l'usager du service social et la plateforme numérique à travers laquelle se font désormais les démarches. Mais en fonction du niveau de soutien des collectivités aux structures où sont employés ces travailleurs sociaux, leur rôle de médiateur, et notamment de médiateur numérique est plus ou moins bien assuré. Le manque de formation et d'équipement des professionnels de l'action sociale dans certaines structures présentes dans les deux quartiers ne permet pas toujours de jouer ce rôle d'alternative à l'accès indépendant aux ressources numériques (notamment l'information administrative).

$\mathrm{Si}$, à LRLB, les personnes interrogées font autant appel à leur réseau social (liens forts, notamment familiaux et liens plus faibles) qu'à TBS, elles ont dans l'ensemble plus aisément recours aux services sociaux pour accéder à des ressources telles qu'un emploi, des revenus (ponctuels ou réguliers), des informations, etc. À TBS, les personnes interrogées font au contraire plus couramment appel aux ressources locales du quartier. Peut-on imputer ces différences aux caractéristiques urbaines des deux quartiers, et en particularité à leur degré d'accessibilité? Avant de répondre positivement à cette question, il importe de souligner que l'insertion généralisée des habitants de LRLB dans le parc de logement social les stabilise, au moins jusqu'à un certain degré, dans un dispositif d'aide sociale, ne serait-ce que par le logement, tandis qu'une partie des personnes interrogées à TBS, en particulier les migrants, présente une fragilité supplémentaire liée à la difficulté à trouver un dispositif d'assistance sociale répondant à leur situation particulière. Démêler, dans les différentes stratégies mises en œuvre par les habitants des deux quartiers, ce qui relève de la contrainte spatiale de ce qui est imputable aux conditions sociales d'existence conduit à se pencher sur la question de l'écologie urbaine au sens où l'entendait Robert Park (1925). L'observation des stratégies mises en œuvre par les plus désaffiliés des enquêtés dans le quartier de TBS confirme le fonctionnement spécifique des quartiers urbains centraux permettant une insertion des migrants grâce à des réseaux d'aide informelle liés à la centralité (Toubon \& Messamah, 1990).

On peut ainsi identifier deux types d'alternatives permettant de s'affranchir de la dépendance numérique : le recours au réseau social, familial, et aux travailleurs sociaux pour accéder aux ressources recherchées également disponibles sur Internet, et l'ancrage local, qui suppose un certain nombre de déplacements pour pallier l'absence d'usage d'Internet. Ces stratégies alternatives à la dépendance numérique se heurtent cependant à des limites pour l'accès aux ressources recherchées. Par exemple, le recours aux 
déplacements de proximité (logique d'ancrage local) sans recherche d'informations préalables sur Internet amène certaines des personnes interrogées à perdre du temps en déplacements inutiles par manque de renseignements sur les horaires ou sur le fonctionnement de certains services administratifs ou sociaux. La logique du recours au réseau social se heurte elle aussi à la faiblesse du capital social d'une partie des personnes interrogées. En fonction du degré d'isolement social des individus, cette stratégie est plus ou moins efficace pour accéder aux ressources. Le renoncement à certaines ressources (culturelles, de loisirs par exemple) que ces personnes savent disponibles sur Internet est ainsi une issue fréquente due à l'absence dans l'entourage de personnes susceptibles de les aider à y accéder (soit que leur réseau social soit trop réduit, soit en raison de la faiblesse des compétences numériques de cet entourage).

L'identification de différentes stratégies d'évitement d'Internet amène à nuancer l'idée d'une dépendance numérique avenue. Les non-usagers d'Internet nous montrent qu'il est encore possible aujourd'hui de formuler et de réaliser des projets résidentiels, professionnels, de s'investir socialement et sentimentalement - ce qui nécessite de mobiliser un certain nombre de ressources diverses - sans avoir recours à Internet. Cependant, ces alternatives, dans la mesure où elles résultent non pas d'un choix, mais de contraintes et d'un habitus freinant l'appropriation d'Internet, ajoutent aux inégalités existantes. De surcroît, pour ces personnes parmi les plus isolées socialement, le nonusage d'Internet est parfois vécu comme une exclusion supplémentaire. Quand la diffusion d'Internet est si massive que la pratique d'activités en ligne n'est plus en soi un marqueur distinctif de certains groupes favorablement placés dans l'espace social, la massification de ces pratiques conduit à une stigmatisation d'autant plus grande des nonutilisateurs dans certaines situations de la vie quotidienne. Ce sentiment de ne pas participer à la «société de l'information" s'exprime au cours des entretiens par l'importance du champ lexical de la dialectique dedans/dehors qui revient dans des expressions comme "c'est pas pour moi » ou «je ne suis pas dedans», toutes énoncées par des personnes de plus de 40 ans. Ces non-usagers mettent en avant leur manque de compétences informatiques et l'absence de besoin d'utiliser Internet pour expliquer cet éloignement de l'outil ${ }^{12}$. Tous les non-usagers âgés de 26 à 35 ans interrogés envisageaient au contraire de s'équiper en informatique, quand ils n'étaient pas dans une situation précaire vis-à-vis de leur logement, et de commencer à utiliser Internet dans un avenir plus ou moins défini. Mais pour ces non-usagers appartenant à la génération précédant tout juste celle qui est née dans un univers massivement informatisé, une déscolarisation précoce a contribué à les éloigner de l'informatique et d'Internet. Plusieurs d'entre eux ne se sont connectés à Internet pour la première fois que par le biais de smartphones pour des usages ludiques (visionner des vidéos). Pour certains, la faiblesse de leurs compétences numériques, à relier à une faible maîtrise de l'écrit, freine leur progression professionnelle jusque dans certains métiers faiblement qualifiés, comme par exemple dans la métallurgie où gagne l'informatique. En somme, la faible appropriation d'Internet par ces jeunes adultes en situation de disqualification sociale ne les empêche pas d'avoir accès au travail et à tout un ensemble de ressources nécessaires dans leur vie quotidienne mais contribue à leur disqualification. 


\section{Conclusion}

38 modifier l'influence de l'espace dans le maintien des inégalités sociales. Si la manière
dont l'espace joue dans la permanence des positionnements sociaux ne fait pas l'objet d'un consensus stabilisé en sciences sociales, l'approche par l'accessibilité aux ressources est utile pour appréhender concrètement ce rôle. Or, la valeur prise au cours de la dernière décennie par les ressources immatérielles échangeables par Internet peut réduire l'importance des contraintes spatiales dans l'accès aux ressources, qui s'exercent avec plus de vigueur sur les personnes défavorisées.

Cependant, tout se passe comme si la frange de la population la plus démunie et pour laquelle Internet pourrait renforcer les capacités ne tirait pas autant d'avantages de cette opportunité que les personnes mieux placées dans l'espace social. Alors qu'une grande partie de la société gagne en flexibilité, en rapidité d'accès aux informations, aux services, compétences de plus en plus exigées sur le marché de l'emploi, les personnes les plus désavantagées sont placées devant une contrainte supplémentaire: subissant, parfois indirectement, une pression sociale pour utiliser Internet, elles sont pourtant confrontées à des difficultés à répondre à cette exigence. Loin de faire vaciller les positionnements sociaux hérités, l'essor d'Internet pourrait au contraire contribuer à fragiliser encore les personnes en situation de disqualification sociale. Pour autant, des expérimentations menées localement ${ }^{13}$ suggèrent que l'appropriation d'Internet par des personnes socialement disqualifiées, dans le cadre d'un projet d'accompagnement centré sur leurs besoins et envies, peut permettre la reconquête de l'estime de soi et favoriser la (ré)insertion sociale. Elles confirment l'importance de l'accompagnement social pour faire d'Internet un outil de renforcement des capacités.

L'observation des modalités individuelles d'accès aux ressources sociales dans deux contextes urbains différents au sein d'une même agglomération conduit à minimiser le rôle du degré d'urbanité dans le niveau de recours à Internet par rapport aux autres modalités d'accès identifiées (recours au réseau social, à l'aide sociale institutionnalisée, ou recours aux ressources locales). La comparaison des stratégies de contournement d'Internet des personnes interrogées dans un quartier central et un quartier périphérique conduit certes à relever certaines différences dans les pratiques, mais attribuer ces pratiques aux caractéristiques urbaines des quartiers concernés reste une opération délicate. Cette complexité illustre bien la difficulté que pose toute tentative de démêler la dimension purement spatiale de toute question sociale. Pour l'heure, il semble que, si Internet vient redistribuer les cartes de ce jeu complexe, il n'en bouleverse pas la configuration. 


\section{BIBLIOGRAPHIE}

BACQUÉ Marie-Hélène \& FOL Sylvie (2007). « Effets de quartier : enjeux scientifiques et politiques de l'importation d'une controverse ", in Authier Jean-Yves, Bacqué Marie-Hélène et Guérin-Pace France (dir.), Le quartier, enjeux scientifiques, actions politiques et pratiques sociales, Paris, La Découverte.

BACQUÉ Marie-Hélène \& FOL Sylvie (2008). « Les politiques de mixité sociale en France : de l'injonction politique nationale aux contradictions locales », in Fée David \& Nativel Corinne (dir.), Crises et politiques du logement en France et au Royaume-Uni, Paris, Presses de la Sorbonne Nouvelle. BEAUDE Boris (2008a). Éléments pour une géographie du lieu réticulaire. Avoir lieu aujourd'hui, Thèse de doctorat en géographie sous la direction de Rémy Knafou, Université de Paris 1.

BEAUDE Boris (2008b). « Internet, lieu du Monde? », in Lévy Jacques (dir.), L'invention du Monde, Paris, Presses de Sciences Po, pp. 111-132.

BIDOU-ZACHARIASEN Catherine (1997). «La prise en compte de "l'effet de territoire" dans l'analyse des quartiers urbains », Revue Française de Sociologie, 38 (1), pp. 97-117.

BOLTANSKI Luc \& CHIAPELLO Eve (1999). Le nouvel esprit du capitalisme, Paris, Gallimard. BOURDIEU Pierre (1993). « Effets de lieu », in La misère du monde, Paris, Seuil, pp. 249-262. BOUTET Annabelle \& TREMEMBERT Jocelyne (2008). Les recherches sur les usages des tic à l'épreuve de la problématique des non-usages d'internet et de l'informatique, document de travail, http:// www.marsouin.org/spip.php?article248 (consulté le $1^{\text {er }}$ juin 2012).

BOUTET Annabelle \& TREMEMBERT Jocelyne (2009). « Mieux comprendre les situations de non-usage des TIC. Le cas d'Internet et de l'informatique. Réflexion méthodologique sur les indicateurs de l'exclusion dite numérique ", Les Cahiers du numérique, 5 (1), pp. 69-100.

CASTELLS Manuel (1996). The Rise of the Network Society. (The Information Age: Economy, Society and Culture, Volume 1), Oxford, Wiley-Blackwell.

CLAVAL Paul (1981). La logique des villes. Essai d'urbanologie, Géographie économique et sociale, 15, Paris, Litec.

CREDOC (2011). La diffusion des technologies de l'information dans la société française, rapport réalisé à la demande du ministère de l'Économie, des Finances et de l'Emploi et de l'ARCEP.

DAGIRAL Éric (2011). « Administration électronique », Communications, 88, pp. 9-17.

DONZELOT Jacques (1991). « Le déplacement de la question sociale », in Jacques Donzelot (dir.), Face à l'exclusion, le modèle français, Paris, Esprit, pp. 5-11.

DUPUY Gabriel (1987). « Vers une théorie territoriale des réseaux : une application au transport urbain », Annales de Géographie, 538, pp. 658-679.

DUPUY Gabriel (2006). La dépendance à l'égard de l'automobile, Paris, La Documentation française. FERREIRA Antonio \& BATEY Peter (2007). « Re-thinking accessibility planning. A multi-layer conceptual framework and its policy implications », Town Planning Review, 78 (4), pp. 429-458. FOL Sylvie (2010). « Encouragement ou injonction à la mobilité ? » Projet, 314 (1), pp. 52-58. 
FOL Sylvie (2005). Mobilité des pauvres et rapports au territoire, Mémoire pour l'habilitation à diriger des recherches, Université de Paris 1 Panthéon-Sorbonne.

FRANÇOIS Jean-Christophe, GRASLAND Claude \& LE GOIX Renaud (2002). « Intégration spatiale et intégration sociale dans la ville : L'espace compte », L'Espace géographique, 31 (4), pp. 355-356.

GRAFMEYER Yves (1995). Sociologie urbaine, Paris, Nathan.

GRANJON Fabien (2010). « Le "non-usage" de l'internet : reconnaissance, mépris et idéologie ", Questions de communication, 18, pp. 37-61.

GRANJON Fabien (2011). « Fracture numérique », Communications, 88, pp. 67-74.

GUICHARD Éric (2002). L'internet : mesures des appropriations d'une technique intellectuelle. Thèse de doctorat sous la direction de Hervé Le Bras, EHESS, Paris, http://www-sop.inria.fr/axis/ personnel/Eric.Guichard/theseEG/ (consulté le $1^{\text {er }}$ juin 2012).

GUICHARD Éric (2011). « Le mythe de la fracture numérique », in Guichard Éric (dir.), L'Internet : Regards croisés, Presses de l'ENSSIB, Villeurbanne, http://barthes.enssib.fr/articles/Guichard-

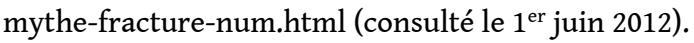

KAIN John (2004). « A Pioneer's Perspective on the Spatial Mismatch Literature », Urban Studies, 41 (1), pp. 7-32.

LENHART Amanda, HORRIGAN John, RAINIE Lee, ALLEN Katherine, BOYCE Angie, MADDEN Mary \& O'GRADY Erin (2003). The ever-shifting internet population: a new look at internet access and the digital divide, Pew Internet and American Life Project, http://www.pewinternet.org/Reports/2003/TheEverShifting-Internet-Population-A-new-look-at-Internet-access-and-the-digital-divide.aspx (consulté le 15 septembre 2012).

LÉVY Jacques (1994). L'espace légitime, Paris, Presses de la fondation nationale des sciences politiques.

L'HORTY Yannick \& SARI Florent (2008). Les zones urbaines sensibles en Île-de-France : typologie des tensions territoriales, Document de travail du CEE, 99/ Document de recherche EPEE, 08-10.

PARK Robert (1925 [2004]). « La ville. Proposition de recherche sur le comportement humain en milieu urbain », in Grafmeyer Yves \& Joseph Isaac (dir.), L'École de Chicago. Naissance de l'écologie urbaine, Paris, Flammarion, pp. 83-130.

PAUGAM Serge (2000). La Disqualification sociale : essai sur la nouvelle pauvreté, Paris, Presses Universitaires de France.

RENAHY Nicolas (2010). « Classes populaires et capital d'autochtonie. Genèse et usages d'une notion ", Regards sociologiques, 40, pp. 9-26.

RIPOLL Fabrice \& TISSOT Sylvie (2010). «La dimension spatiale des ressources sociales », Regards sociologiques, 40, pp. 5-7.

RIPOLL Fabrice \& VESCHAMBRE Vincent (2005). « Sur la dimension spatiale des inégalités :

contribution aux débats sur la "mobilité" et le "capital spatial” ", in Arlaud Samuel, Jean Yves \& Royoux Dominique (dir.), Rural-Urbain. Nouveaux liens, nouvelles frontières, Rennes, PUR, pp. 467-483.

SOJA Edward W. (2009). « Taking space personaly », in Warf Barney \& Arias Santa (dir.), The Spatial Turn: Interdisciplinary Perspectives, Abingdon: New York, Routledge, pp. 11-35.

TOUBON Jean-Claude \& MESSAMAH Khelifa (1990). Centralité immigrée. Le quartier de la Goutte d'Or, Paris, L'Harmattan. 
WARF Barney \& ARIAS Santa (dir.) (2009), The Spatial Turn: Interdisciplinary Perspectives, Abingdon: New York, Routledge.

\section{NOTES}

1. L'accessibilité des lieux peut elle-même se comprendre comme une mesure de leur dotation en multiples ressources et de la facilité avec laquelle on peut, depuis ces lieux, atteindre des ressources localisées en d'autres lieux.

2. Il faut bien entendu nuancer ce constat d'un renforcement avec le temps de la spécialisation sociale des quartiers : sous l'effet des politiques publiques mais également de jeux d'acteurs à des échelles multiples, certains quartiers populaires connaissent une revalorisation économique et symbolique qui modifie profondément leur structure sociale (en particulier certains quartiers centraux et péricentraux des grandes villes perdent leur caractère populaire), d'autres subissent une dévalorisation qui affecte également leur structure sociale par une fuite des populations les plus aisées. Si l'intervention publique s'inscrit traditionnellement dans la recherche d'une homogénéisation, notamment sociale, des morceaux de la ville, les actions menées en ce sens conduisent parfois à orienter la structure sociale d'un quartier vers une nouvelle spécialisation (Bacqué \& Fol, 2008).

3. L'acronyme se réfère généralement aux "Technologies» de l'Information et de la Communication. On préfèrera utiliser ici le terme de "technique " plutôt que celui de « technologie » qui fait référence à la théorie des techniques. La confusion usuelle entre les deux termes s'explique par une survalorisation des techniques «de pointe ", à haute valeur ajoutée, connotée par le terme de «technologie » dans le langage courant (Guichard, 2002).

4. La dénomination de ce domaine de l'action publique est trompeuse puisqu'elle semble désigner l'action en faveur de l'ensemble de la ville, alors que la Politique de la Ville n'est dirigée que vers certains quartiers spécifiques.

5. Pour intégrer à la question de l'accessibilité ce type de mécanismes par lequel l'espace joue, il reste cependant à examiner les modes d'accès à cette ressource : le capital d'autochtonie est-il reçu en héritage par le simple fait d'une naissance en un lieu ou bien y accède-t-on par accumulation de preuves d'appartenance à un territoire, elles-mêmes susceptibles d'être décomposées en multiples ressources?

6. Les entretiens ont été conduits pour la plupart en 2008 et 2009. Certains d'entre eux ont été prolongés par un entretien complémentaire en 2011 afin de suivre les évolutions de ces différentes trajectoires.

7. Je ne compte pas ici les deux quartiers de la commune de Rive-de-Gier, signataire également du CUCS, mais située dans une autre agglomération selon la définition de l'INSEE.

8. Au sein de l'association Alpha (association d'alphabétisation et d'apprentissage du français) à Beaubrun, du collectif chômeur de l'Ondaine, et du Centre Social La Mosaïque à LRLB.

9. La dépendance automobile est une configuration sociale dans laquelle tout est prévu pour le déplacement en voiture (voir Dupuy, 2006).

10. On pourrait parler pour être plus précis de "groupe d'amis » reliant des personnes de la même génération et du même sexe, en l'occurrence de jeunes hommes. Cependant, certainement en raison d'une certaine pudeur, les jeunes hommes considérés parlent plus volontiers d'un groupe de "collègues ». Précisons que, dans le langage courant des jeunes stéphanois, et plus généralement des jeunes rhônalpins, le terme de "collègue " ne désigne pas une connaissance professionnelle, mais toute personne avec qui on partage une activité sociale. Il est un synonyme, légèrement euphémique, de « copain ». 
11. Il s'agit principalement des personnes dont la mobilité est freinée par des capacités physiques moindres (pour les personnes âgées ou souffrant de handicaps), par des programmes d'activités particulièrement contraints (pour les mères de familles monoparentales, par exemple).

12. L'expression d'une absence de besoin d'utiliser Internet est un indicateur que "l'effet de club», ou "effet de réseau", qui consiste à voir la valeur d'usage d'un mode de télécommunication augmenter pour un individu au fur et à mesure que les membres de son réseau social s'en emparent, n'a pas joué. La force du réseau social d'un individu est un déterminant essentiel de l'appropriation d'Internet. C'est également la conclusion à laquelle parviennent les membres du GIS (Groupement d'Intérêt Scientifique) Marsouin à partir de leur recherche sur l'e-inclusion (voir en particulier Boutet et Tremembert, 2009)

13. Les divers projets mis en œuvre par la Ville de Brest dans le domaine du multimédia et de l'insertion sociale (présentés sur www.reperes-brest.net et sur http://www.a-brest.net/ article7535.html) en sont un exemple significatif.

\section{RÉSUMÉS}

Dans quelle mesure la diffusion d'Internet modifie-t-elle les contraintes spatiales et temporelles qui s'exercent sur les activités humaines? Réduit-elle l'inégale répartition des ressources sur les territoires et surtout l'inégale accessibilité à ces ressources? Dans une démarche d'observation participante couplée à des entretiens, deux quartiers d'une même agglomération placés en zone urbaine sensible, l'un central et l'autre périphérique, ont été étudiés : l'article décrit comment leurs habitants se sont appropriés ou non Internet dans la réalisation de certains actes quotidiens, comme la recherche d'emploi par exemple, pour discuter des possibles effets du média sur l'inscription spatiale des inégalités sociales. L'article commence par revenir sur l'approche géographique des inégalités, rappelant la nécessaire dénaturalisation de la répartition spatiale des catégories sociales et explicitant en particulier la notion d'accessibilité. La manière dont les nouvelles technologies et Internet en particulier viennent bousculer ces problématiques, puisqu'elles modifient les conditions d'accès à certaines ressources, sera ensuite précisée, de même que le protocole d'enquête utilisé ici pour mesurer la réalité de ces transformations. Si l'analyse des résultats révèle alors des comportements en évolution - assez semblables d'ailleurs entre les deux quartiers - et même l'existence de stratégies, chez les non-usagers d'Internet, pour s'affranchir de la dépendance numérique, les inégalités spatiales tendent plutôt à se consolider, nouvelle preuve de la cumulativité des différentes formes d'inégalités.

To what extent does the spread of Internet uses change space and time constraints affecting human activities? Is it reducing the unequal distribution of resources between territories, and above all the unequal accessibility to these resources? The residents of two neighborhoods considered as 'urban renewal zones' ('zones urbaines sensibles') of the same city area, a central one and another peripheral, have been surveyed through participant observation and interviews. The article describes how the Internet has been seized (or not) by their inhabitants in the fulfillment of everyday activities such as job searching, in order to discuss the possible effects of the media on how social inequalities are spatially embedded. The article first presents the geographical approach of inequalities, reminding that it is necessary to consider the spatial distribution of social inequalities as unnatural, and clarifies the notion of accessibility. The way new technologies and the Internet in particular disrupt this kind of issues, since they modify the 
conditions of access to several resources, will then be specified, as well as the research protocol used in this case to assess the reality of theses transformations. Finally, the analysis reveals changing behaviors, that follow similar patterns in both neighborhoods, and the existence of strategies among the Internet non-users too, in order to overcome digital dependency. However, spatial inequalities are rather consolidating, an additional evidence of the cumulativity of inequalities.

INDEX

Mots-clés : inégalités socio-spatiales, usages et non-usages d'Internet, accessibilité, ressources sociales, quartiers défavorisés

Keywords : social and spatial inequalities, Internet uses, accessibility, social resources, deprived urban areas

\section{AUTEUR}

\section{MARGOT BEAUCHAMPS}

Université Paris 1 Panthéon-Sorbonne, UMR Géographie-Cités, équipe CRIA 